PROCEEDINGS OF THE

AMERICAN MATHEMATICAL SOCIETY

Volume 132, Number 2, Pages 347-352

S 0002-9939(03)07153-3

Article electronically published on June 23, 2003

\title{
UNIQUENESS OF EXCEPTIONAL SINGULAR QUARTICS
}

\author{
KAREN A. CHANDLER
}

(Communicated by Michael Stillman)

\begin{abstract}
We prove that given a general collection $\Gamma$ of 14 points of $\mathbb{P}^{4}=\mathbb{P}_{\mathcal{K}}^{4}$ $(\mathcal{K}$ an infinite field) there is a unique quartic hypersurface that is singular on $\Gamma$.

This completes the solution to the open problem of the dimension of a linear system of hypersurfaces of $\mathbb{P}^{n}$ that are singular on a collection of general points.
\end{abstract}

\section{INTRODUCTION}

Let $\mathcal{K}$ be an infinite field and $\mathbb{P}^{n}=\mathbb{P}_{\mathcal{K}}^{n}$.

The following problem has aroused a good deal of interest over the last few centuries:

Question 1. Let $\Gamma$ be a general set of $d$ points in $\mathbb{P}^{n}$. Given a degree $m \geq 3$, does the vector space of sections in $H^{0}\left(\mathbb{P}^{n}, \mathcal{O}_{\mathbb{P}^{n}}(m)\right)$ that are singular on $\Gamma$ have the expected dimension of $\max \left(0, \operatorname{dim} H^{0}\left(\mathbb{P}^{n}, \mathcal{O}_{\mathbb{P}^{n}}(m)\right)-(n+1) d\right)$ ?

The answer is that the only exceptions are the following 4 cases: $(n, m, d)=$ $(2,4,5),(3,4,9),(4,4,14)$, and $(4,3,7)$. This was proved by J. Alexander and A. Hirschowitz $([\mathrm{H}], \mathrm{A}], \mathrm{AH}]$, AH2], and $\underline{\mathrm{AH}}]$ ). (A simpler proof was later given in Ch2 and Ch3].)

A correspondence between the question on singularities and the Waring problem for general linear forms was (for char $\mathcal{K}=0$ ) described by Lasker [L]. Terracini [T2] applied the duality of Macaulay to make this precise. Terracini [T1], as well as Palatini $[\mathrm{P}]$, gave a further relation to the study of a secant variety to a Veronese. (See [EI] for an extension to char $\mathcal{K} \neq 0$.) The Waring problem asks: given $n, m$, what is the minimal $d=(n, m)$ for which the general form of degree $m$ in $n+1$ variables may be written as a sum of $d m$ th powers of linear forms? The expectation is that $(n+1) d \geq\left(\begin{array}{c}n+m \\ m\end{array}\right)$ should suffice (since there are $d$ choices from the $(n+1)$ dimensional space of linear forms). The exceptional case of $(n, m, d)=(2,4,5)$ was discovered by Clebsch [C], followed by those of $(3,4,9),(4,4,14)$ due to Sylvester $[$ ], and the more subtle case of $(4,3,7)$ presented by Palatini $[\mathrm{P}$.

In each of the exceptional cases we have $(n+1) d \geq \operatorname{dim} H^{0}\left(\mathbb{P}^{n}, \mathcal{O}\left(\mathbb{P}^{n}(m)\right)\right.$ hence no $m$-ic form is "numerically" expected to be singular at a general collection of $d$ points. However, one may easily find such an $m$-ic in each of these cases. We consider, therefore, the question of the "next best" possibility:

Received by the editors April 17, 2001 and, in revised form, October 14, 2002.

2000 Mathematics Subject Classification. Primary 14N10; Secondary 14C20.

(C)2003 American Mathematical Society 
Question 2. In the exceptional cases, is there a unique $m$-ic singular along $d$ general points?

The affirmative answer for the case of 7 points in $\mathbb{P}^{4}$ and degree 3 was given by C. Ciliberto and Hirschowitz [CH]. This is discussed, e.g., in [Ch2].

We consider the exceptional cases in degree 4 , namely, 5 points in $\mathbb{P}^{2}, 9$ in $\mathbb{P}^{3}$, and $14 \mathrm{in} \mathbb{P}^{4}$. In each, $d=\operatorname{dim} H^{0}\left(\mathbb{P}^{n}, \mathcal{O}_{\mathbb{P}^{n}}(2)\right)-1$, so that there is a quadric $Q$ vanishing on $d$ general points, hence $Q^{2}$ is singular at each point. Thus we show that $Q^{2}$ is the only such quartic. J. Alexander proves this in the cases of $\mathbb{P}^{2}$ and $\mathbb{P}^{3}$ in $\left[\mathrm{A}\right.$. To obtain uniqueness in $\mathbb{P}^{4}$ we use both of these cases together with a Horace differential argument. This is unlike the usual application of the "méthode d'Horace" in which a codimension 1 result suffices in carrying out the induction. The result is:

Theorem 3. If $(n, d)=(2,5),(3,9)$, or $(4,14)$, there is a unique quartic of $\mathbb{P}^{n}$ that is singular on $d$ general points of $\mathbb{P}^{n}$.

Corollary 4. Suppose that char $\mathcal{K} \neq 2$. Take $(n, d)=(2,5),(3,9)$, or $(4,14)$. In the space of homogeneous forms of degree 4 in $n+1$ variables, the closure of the set of those expressible as a sum of d fourth powers of linear forms has codimension 1.

Corollary 5. Given $n, m, d$, let $N=\left(\begin{array}{c}n+m \\ m\end{array}\right)-1$. Let $\nu_{m}: \mathbb{P}^{n} \rightarrow \mathbb{P}^{N}$ be the mth Veronese embedding of $\mathbb{P}^{n}$. Call $S_{n, m, d}$ the variety of secant $(d-1)$-planes to $\nu_{m}\left(\mathbb{P}^{n}\right)$ in $\mathbb{P}^{N}$. Then for $(n, d)=(2,5),(3,9)$, or $(4,14), S_{n, m, d}$ is a hypersurface of $\mathbb{P}^{N}$.

Let us recall standard definitions in the study of such objects:

Definition 1. Let $p \in \mathbb{P}^{n}$. The double point at $p$ in $\mathbb{P}^{n}$ is the subscheme of $\mathbb{P}^{n}$ defined by the square of the ideal sheaf of $p$.

If $\Phi \subset \mathbb{P}^{n}$, we denote by $\Phi^{2}$ the union of the double points supported on $\Phi$.

Hence a homogeneous form in the coordinate ring of $\mathbb{P}^{n}$ is singular on a set $\Phi$ precisely if it vanishes on $\Phi^{2}$.

Definition 2. Given a scheme $X \subset \mathbb{P}^{n}$ and a hyperplane $H$ of $\mathbb{P}^{n}$, the Castelnuovo exact sequence is given by

$$
0 \rightarrow \mathcal{I}_{\tilde{X}}(-1) \rightarrow \mathcal{I}_{X} \rightarrow \mathcal{I}_{X \cap H, H} \rightarrow 0,
$$

where $\tilde{X}$ (called the residual scheme to $X$ with respect to $H$ ) is given by the ideal sheaf $\mathcal{I}_{\tilde{X}}=\mathcal{I}_{X}: \mathcal{O}_{\mathbb{P}^{n}}(-H)$.

From this, it is straightforward to prove the uniqueness in $\mathbb{P}^{2}$ and $\mathbb{P}^{3}$ using specialisation, as is done in $\left[\mathrm{A}\right.$. But in $\mathbb{P}^{4}$ the exact sequence reveals only that there is at most a pencil of quartics through 14 double points. This is because the case of $\mathbb{P}^{3}$ is extra-exceptional: although $4 \cdot 9>\operatorname{dim} H^{0}\left(\mathbb{P}^{3}, \mathcal{O}_{\mathbb{P}^{3}}(4)\right)$, there is a quartic singular on 9 general points. Hence the base locus of the system of quartics singular on 8 double points and a point $q$ meets the double point at $q$ in a scheme $\rho$ of degree 3. Applying Castelnuovo to a suitable collection $Z \subset \mathbb{P}^{4}$ of 13 double points, of which 8 lie on a $\mathbb{P}^{3}$ containing a point $q$, shows that the base locus of quartics through $Z \cup\{q\}$ meets $\{q\}^{2}$ in the scheme $\rho$ determined by those 8 points on $\mathbb{P}^{3}$. So $Z \cup\{q\}^{2}$ lies on a pencil of quartics.

To conquer this obstacle, we apply the lemme d'Horace différentielle (Lemma [6) of Alexander and Hirschowitz ( $\overline{\mathrm{AH} 1})$. The statement extracted from the lemma 
is that from such a scheme $Z \cup\{q\}^{2}$ lying on a pencil of quartics together with base locus scheme $\rho$, one may find a point $p$ for which $Z \cup\{p\}^{2}$ is on a unique quartic provided that $\tilde{Z} \cup \rho$ does not lie on a cubic. The idea is to degenerate a point $p \in \mathbb{P}^{4}-\mathbb{P}^{3}$ to $q$ along with a subscheme $\rho^{\prime} \subset\{p\}^{2}$ degenerating to $\rho$. Hence the base locus of quartics through $Z \cup\{p\}$ meets $\{p\}^{2}$ in a subscheme of $\rho^{\prime}$. But then, Castelnuovo's exact sequence may be applied directly to $Z \cup \rho^{\prime}$, to see that if $\tilde{Z} \cup \rho^{\prime}$ does not lie on a cubic, then the base locus of quartics through $Z \cup\{p\}$ cannot contain all of $\rho^{\prime}$. Hence by upper semicontinuity it suffices that $\tilde{Z} \cup \rho$ does not lie on a cubic.

The uniqueness in $\mathbb{P}^{4}$ is therefore accomplished by producing such a scheme $Z \cup$ $\{q\}$ along with base locus scheme $\rho$ determined by $Z \cap \mathbb{P}^{3}$ for which $\tilde{Z} \cup \rho$ does not lie on a cubic. Just as well, we arrange that $\rho$ has a subscheme $\rho_{0}$ of degree 2 whose union with $\tilde{Z}$ does not lie on a cubic. Hence it is desired to have some control over the base locus scheme $\rho$ at $q$. For this we arrange by further specialisation (analogous to $\mathrm{Ch} 2$, in the initial case of 12 points in $\mathbb{P}^{5}$ ) that $\rho$ has a recognizable such subscheme $\rho_{0}$ that does not depend on all the points. Namely, 4 of the points of $Z \cap \mathbb{P}^{3}$ are put onto a plane containing $q$, so that the base locus scheme $\rho$ must contain the degree 2 scheme $\rho_{0}$ on $q$ given by the conic through the 5 planar points.

Hence the problem is reduced to a matter of studying cubics on the union of 5 general double points, 4 simple points on $\mathbb{P}^{3}$ (and otherwise set free), with a degree 6 curvilinear subscheme of $\mathbb{P}^{2}$ (in linearly general position). Now the four simple points may be further specialised to $\mathbb{P}^{2}$, yielding $\mathbb{P}^{2}$ in the base locus. Then it is easy to see that no cubic of $\mathbb{P}^{4}$ vanishes on the general union of $\mathbb{P}^{2}$ with five double points, which completes the proof.

Notation. For a subscheme $X \subset \mathbb{P}^{n}$, we write $h_{\mathbb{P}^{n}}(X, m)$ for the Hilbert function of $X$ in degree $m$ : the number of conditions that $X$ imposes on the linear system of hypersurfaces of degree $m$.

Taking global sections on the Castelnuovo exact sequence (11) then provides the inequality:

$$
h_{\mathbb{P}^{n}}(X, m) \geq h_{\mathbb{P}^{n}}(\tilde{X}, m-1)+h_{H}(X \cap H, m)
$$

where $H$ is a hyperplane and $\tilde{X}$ the residual of $X$ with respect to $H$.

\section{Proof of Theorem 3}

Fix a flag $\mathbb{P}^{2} \subset \mathbb{P}^{3} \subset \mathbb{P}^{4}$.

We show that there is a unique quartic hypersurface of $\mathbb{P}^{4}$ through the union of 14 general double points. To do this, we construct a scheme from the ground up, collecting subschemes with support on $\mathbb{P}^{2}$ and on $\mathbb{P}^{3}$ and thereby observing uniqueness in dimensions 2 and 3 along the way.

Dimension 2. Suppose that $\Psi \cup\{q\} \subset \mathbb{P}^{2}$ is a set of 5 points, no three of which are collinear. So $\Psi \cup\{q\}$ lies on a unique conic $C$ (nonsingular and irreducible) defined by a quadric form $Q$. Suppose $F$ is a quartic form vanishing on $\Phi^{2}$. Then $F$ vanishes on a subscheme of $C$ of degree 10 , hence $Q \mid F$, say $F=G \cdot Q, \operatorname{deg} G=2$. Then $G$ also vanishes on $\Psi \cup\{q\}$ (since $C$ is nonsingular); so, up to constants, we have $G=Q$ and $F=Q^{2}$. Hence we have uniqueness.

Notice, in particular, that the base locus of quartics through $\Psi^{2} \cup\{q\}$ meets $\{q\}^{2}$ in precisely $\{q\}^{2} \cap C$. 
Dimension 3. Let $\Phi \subset \mathbb{P}^{3}-\mathbb{P}^{2}$ be a set of 4 points in linearly general position. Then it is easy to see (e.g. straight from the ideal) that

$$
h_{\mathbb{P}^{3}}\left(\Phi^{2}, 3\right)=16
$$

and

$$
h_{\mathbb{P}^{3}}\left(\Phi^{2} \cup \mathbb{P}^{2}, 3\right)=20
$$

(i.e., $\Phi^{2}$ does not lie on a quadric). So we may find a (general) set $\Psi \subset \mathbb{P}^{2}$ of 4 points so that $\Phi^{2} \cup \Psi$ does not lie on a cubic. Now choose $q \in \mathbb{P}^{2}$ so that $\Psi \cup\{q\}$ is in linearly general position (with respect to $\left.\mathbb{P}^{2}\right)$. Then $\left(\Psi^{2} \cup\{q\}^{2}\right) \cap \mathbb{P}^{2}$ lies on a unique quartic of $\mathbb{P}^{2}$. Hence by (1) there is a unique quartic that is singular on the collection $\Phi \cup \Psi \cup\{q\}$ of 9 points of $\mathbb{P}^{3}$.

Further,

$$
h_{\mathbb{P}^{2}}\left(\Psi^{2} \cup\{q\}, 4\right)=13
$$

SO

$$
h_{\mathbb{P}^{3}}\left(\Phi^{2} \cup \Psi^{2} \cup\{q\}, 3\right) \geq 20+14=4 \cdot 8+1,
$$

so that equality holds here. Therefore the system of quartics through $\Phi^{2} \cup \Psi^{2} \cup\{q\}$ has base locus meeting $\{q\}^{2}$ in precisely a scheme $\rho$ of degree 3 .

Let $C$ be the conic through $\Psi \cup\{q\}$ in $\mathbb{P}^{2}$ and $\rho_{0}=\{q\}^{2} \cap C$. As we have seen, $\rho_{0} \subset \rho$.

Dimension 4. Take $\Phi \subset \mathbb{P}^{3}, \Psi \cup\{q\} \subset \mathbb{P}^{2}, \rho_{0}, \rho$ just as in the case of dimension 3 . Consider a set $\Sigma \subset \mathbb{P}^{4}-\mathbb{P}^{3}$ of 5 points in linearly general position and $Z=$ $\Sigma^{2} \cup \Phi^{2} \cup \Psi^{2}$.

We apply the following:

Lemma 6 ([AH1]). Choose a hyperplane $H \subset \mathbb{P}^{n}$. Let $X \subset \mathbb{P}^{n}$ be a union of double and simple points of $\mathbb{P}^{n}$ and $\tilde{X}$ its residual with respect to $\mathbb{P}^{n-1}$. Let $\Upsilon$ be a subscheme of a double point supported at a point $q \in H$.

Assume that:

- $\operatorname{deg} X \cup \Upsilon=\left(\begin{array}{c}n+m \\ m\end{array}\right)$,

- $(X \cup \Upsilon) \cap H$ does not lie on an $m$-ic of $H$, and

- if $\rho$ is the intersection of $\Upsilon \cap H$ with the base locus of $m$-ics through $(X \cup$ $\{q\}) \cap H$, then $\tilde{X} \cup \rho$ does not lie on an $(m-1)$-ic of $\mathbb{P}^{n}$.

Then there is a translation $\Upsilon^{\prime}$ of $\Upsilon$ so that $X \cup \Upsilon^{\prime}$ does not lie on an $m$-ic hypersurface of $\mathbb{P}^{n}$.

To use the lemma, let us start by taking a general point $r \in \mathbb{P}^{3}$ so that $(Z \cup\{r\} \cup$ $\left.\{q\}^{2}\right) \cap \mathbb{P}^{3}$ does not lie on a quartic (by the uniqueness in $\mathbb{P}^{3}$ ) and set $X=Z \cup\{r\}$.

Next, let us choose $\Upsilon \subset\{q\}^{2} \subset \mathbb{P}^{4}$ of degree 4 and satisfying $\Upsilon \cap \rho=\rho_{0}$ (so $\operatorname{deg} X \cup \Upsilon=70)$. Then $(X \cup \Upsilon) \cap \mathbb{P}^{3}$ does not lie on a quartic of $\mathbb{P}^{3}$ (by virtue of the choice $\rho \not \subset \Upsilon)$. The base locus of quartics through $(X \cup\{q\}) \cap \mathbb{P}^{3}$ then meets $\Upsilon$ in precisely $\rho_{0}$. Hence in order to apply Lemma [6 to $X$ and $\Upsilon$, we see that the scheme $\tilde{X} \cup \rho_{0}=\Sigma^{2} \cup \Phi \cup \Psi \cup \rho_{0}$ does not lie on a cubic.

We have $\Psi \cup \rho_{0} \subset \mathbb{P}^{2}$ and $h_{\mathbb{P}^{2}}\left(\Psi \cup \rho_{0}, 3\right)=6$ ( since $\left.h_{\mathbb{P}^{2}}(C, 3)=7>6\right)$.

Since $\rho_{0}$ does not depend on $\Phi$ we may degenerate $\Phi$ to a set $\Phi_{0} \subset \mathbb{P}^{2}$, so that

$$
h_{\mathbb{P}^{2}}\left(\Phi_{0} \cup \Psi \cup \rho_{0}, 3\right)=10 ;
$$

that is, no cubic of $\mathbb{P}^{2}$ vanishes on $\Phi_{0} \cup \Psi \cup \rho_{0}$. 
Consider, then, a set of 5 points $\Sigma \subset \mathbb{P}^{4}$. If $\Sigma^{2} \cup \mathbb{P}^{2}$ does not lie on a cubic of $\mathbb{P}^{4}$, then neither does $\Sigma^{2} \cup \Phi_{0} \cup \Psi \cup \rho_{0}$, and hence by upper semicontinuity $\Sigma^{2} \cup \Phi \cup \Psi \cup \rho_{0}$ is not on a cubic, as desired.

Thus we are left with finding $\Sigma$, so that $h_{\mathbb{P}^{4}}\left(\Sigma^{2} \cup \mathbb{P}^{2}, 3\right)=35$.

Let us take $\Sigma \subset \mathbb{P}^{4}-\mathbb{P}^{3}$ to be a set of 5 points in linearly general position.

Then $\Sigma^{2}$ does not lie on a quadric of $\mathbb{P}^{4}$, that is,

$$
h_{\mathbb{P}^{4}}\left(\Sigma^{2}, 2\right)=15 .
$$

Further, any cubic through $\Sigma^{2}$ must vanish on the union $\operatorname{Sec} \Sigma$ of lines between pairs of points of $\Sigma$. We have (see, e.g., Ch1])

$$
h_{\mathbb{P}^{3}}\left(\operatorname{Sec} \Sigma \cap \mathbb{P}^{3}, 3\right)=10 .
$$

Hence

$$
\begin{aligned}
h_{\mathbb{P}^{n}}\left(\Sigma^{2} \cup \mathbb{P}^{2}, 3\right) & =h_{\mathbb{P}^{n}}\left(\Sigma^{2} \cup \operatorname{Sec} \Sigma \cup \mathbb{P}^{2}, 3\right) \\
& \geq h_{\mathbb{P}^{4}}\left(\Sigma^{2}, 2\right)+h_{\mathbb{P}^{3}}\left(\left(\operatorname{Sec} \Sigma \cap \mathbb{P}^{3}\right) \cup \mathbb{P}^{2}, 3\right) \\
& \geq h_{\mathbb{P}^{4}}\left(\Sigma^{2}, 2\right)+h_{\mathbb{P}^{3}}\left(\operatorname{Sec} \Sigma \cap \mathbb{P}^{3}, 3\right)+h_{\mathbb{P}^{2}}\left(\mathbb{P}^{2}, 3\right) \\
& =15+10+10=35 .
\end{aligned}
$$

By Lemma 6 there is a point $p \in \mathbb{P}^{4}$ for which

$$
h_{\mathbb{P}^{4}}\left(\Sigma^{2} \cup \Phi^{2} \cup \Psi^{2} \cup\{p\}^{2} \cup\{r\}, 3\right)=70 .
$$

Thus, there is a unique quartic of $\mathbb{P}^{4}$ that is singular on the collection $\Sigma \cup \Phi \cup$ $\Psi \cup\{p\}$ of 14 points.

\section{REFERENCES}

[A] J. Alexander, Singularités imposables en position générale aux hypersurfaces de $\mathbb{P}^{n}$, Compositio Math. 68 (1988), 305-354. MR 89k:14085

[AH1] J. Alexander and A. Hirschowitz, Un lemme d'Horace différentiel: application aux singularités hyperquartiques de $\mathbb{P}^{5}$, J. Algebraic Geom. 1 (1992), no. 3, 411-426. MR 93e:14004

[AH2] J. Alexander and A. Hirschowitz, La méthode d'Horace éclatée: application à l'interpolation en degré quatre, Invent. Math. 107, 585-602 (1992). MR 93d:13017

[AH3] J. Alexander and A. Hirschowitz, Polynomial interpolation in several variables, J. Algebraic Geom. 4 (1995), no. 2, 201-222. MR 96f:14065

[Ch1] K. Chandler, Geometry of dots and ropes, Trans. Amer. Math. Soc. 347 (1995), no. 3, 767-784. MR 95f:14054

[Ch2] K. Chandler, A brief proof of a maximal rank theorem for generic double points in projective space, Trans. Amer. Math. Soc. 353 (2001), no. 5, 1907-1920. MR 2002i:14046]

[Ch3] K. Chandler, Linear systems of cubics singular at generic points of projective space, Compositio Math. 134 (2002), no. 3, 269-282.

[CH] C. Ciliberto and A. Hirschowitz, Hypercubiques de $\mathbb{P}^{4}$ avec sept points singuliers génériques, C. R. Acad. Sci. Paris Sér. I Math. 313 (1991), no. 3, 135-137. MR 92g:14043

[C] A. Clebsch, Über Curven vierter Ordnung, Journal für die Reine und Angewandte Mathematik (Crelle), Bd. 59 (1861), 125-145.

[EI] J. Emsalem and A. Iarrobino, Inverse system of a symbolic power I, J. Algebra 174 (1995), 1080-1090. MR 96i:13017

[H] A. Hirschowitz, La méthode d'Horace pour l'interpolation à plusieurs variables, Manuscripta Math. 50 (1985), 337-388. MR 86j:14013

[IK] A. Iarrobino and V. Kanev, Power sums, Gorenstein algebras, and determinantal loci. Appendix C by Iarrobino and Steven L. Kleiman. Lecture Notes in Mathematics, 1721. Springer-Verlag, Berlin, 1999. MR 2001d:14056

[L] E. Lasker, Zur Theorie die kanonischen Formen, Math. Annalen 58 (1904), 434-440.

$[\mathrm{P}]$ F. Palatini, Sulla rappresentazione delle forme ed in particolare della cubica quinaria con la somma di potenze di forme lineari,. Atti Acad. Torino 38, (1903), 43-50. 
[S] J. J. Sylvester, Sur une extension d'un théorème de Clebsch relatif aux courbes du quatrième degré. Comptes Rendus CII. (1886) 1532-1534.

[T1] A. Terracini, Sulle $V_{k}$ per cui la varieta degli $S_{h}(h+1)$-seganti ha dimensione minore dell'ordinario, Rend. Circ. Mat. Palermo 31 (1911), 527-530.

[T2] A. Terracini, Sulla rappresentazione delle coppie di forme ternarie mediante somme di potenze di forme lineari, Annali di Mat. Pura Appl. Serie III, 24 (1915), 1-10.

Department of Mathematics, University of Notre Dame, Notre Dame, Indiana 46556

E-mail address: kchandle@noether.math.nd.edu 